\section{Advanced endoscopic imaging using narrow-band imaging for diagnosis of Whipple's disease}

Various systemic and gastrointestinal diseases cause mucosal alterations of the duodenum. These include celiac disease, Crohn's disease, congenital enteropathies (e.g. microvillous inclusion disease, tufting enteropathy), and Whipple's disease [1 - 3]. Thus careful endoscopic inspection and targeted biopsies are strongly recommended to achieve a correct diagnosis and avoid false reassurance.

Here we describe the case of a 45-year-old man who presented with diarrhea, weight loss, and mild abdominal pain. In addition, he complained of strong joint pains. Physical examination was unremarkable. Laboratory investigations showed hemoglobin of $8.2 \mathrm{~g} / \mathrm{dL}$ (reference $13-17$ ), iron of $25 \mu \mathrm{g} / \mathrm{dL}(40-160)$, and C-reactive protein of $84 \mathrm{mg} / \mathrm{L}(<5)$. Upper-gastrointestinal endoscopy revealed pale yellow shaggy mucosa with intermittent, superficial, erythematous eroded patches of the duodenum $(\bullet$ Fig. 1 a).

Optical chromoendoscopy with narrowband imaging (NBI) (Olympus, Tokyo, Japan) showed the intestinal villi to appear edematous and slightly flattened. Although no changes of the microvasculature were observed, the villous lymphatics were clearly enhanced (i.e. villous lymphangiectasia) ( $\bullet$ Fig. 1b). Histopathological examination of the intestinal biopsies showed multiple periodic acidSchiff (PAS)-positive macrophages within the lamina propria, characteristic of Whipple's disease ( $\bullet$ Fig. 1 c). Our patient was put on oral antimicrobial therapy with co-trimoxazole for 2 years and remained in remission at 6-month followup.

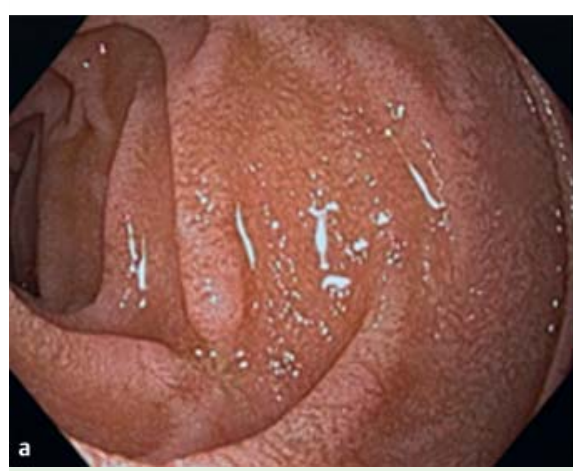

Whipple's disease is a rare multisystemic bacterial infection caused by Tropherymawhipplei that can involve the gastrointestinal, nervous, and cardiac system and the skin [1 - 3]. Previously, we have shown that high-magnification endoscopy can clearly help to visualize the mucosal characteristics typical for Whipple's disease, including edematous and engorged duodenal villi filled with a white material with the appearance of lymph [2]. Although various studies have described the potential of advanced endoscopic imaging in the small bowel, there are currently no data available on the usefulness of chromoendoscopy (either vital or virtual) for the evaluation and diagnosis of Whipple's disease $[3,4]$.

Our case is interesting for several reasons, because it shows for the first time the findings of Whipple's disease using NBI. NBI can aid in the characterization of the mucosal alterations present in Whipple's disease, including villi architecture disarrangement and altered lymphatic microvasculature. In addition, our case highlights clinical and histological characteristics of Whipple's disease. Physicians should be aware of the differential diagnosis and should also consider duodenal biopsies in patients submitted because of extraintestinal symptoms such as joint pains and pericarditis, and increased inflammatory reactions such as elevated sedimentation rate and thrombocytosis [2-4].

\section{Endoscopy_UCTN_Code_CCL_1AC_2AH}

\section{Competing interests: None}

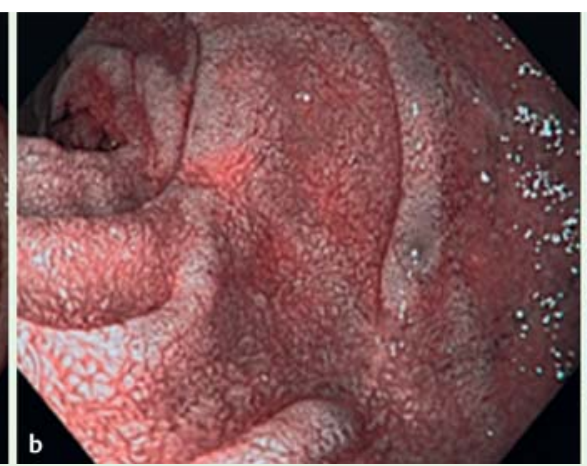

\section{H. Neumann ${ }^{1}$, K. Mönkemüller ${ }^{2}$,}

M. F. Neurath ${ }^{1}$, M. Vieth ${ }^{3}$

1 Department of Medicine I, University of Erlangen-Nuremberg, Erlangen, Germany

2 Department of Internal Medicine and Gastroenterology, Marienhospital Bottrop, Germany

3 Institute of Pathology, Klinikum Bayreuth, Germany

\section{References}

1 Donowitz M, Kokke FT, Saidi R. Evaluation of patients with chronic diarrhea. N Engl J Med 1995; 332: $725-759$

2 Mönkemüller K, Fry LC, von Arnim U et al. Whipple's disease: an endoscopic and histologic study. Digestion 2008; 77: 161 - 165

3 Mönkemüller K, Neumann H, Fry LC. Endoscopic examination of the small bowel: from standard white light to confocal endomicroscopy. Clin Gastroenterol Hepatol 2009; 7: $11-12$

4 Neumann H, Fry LC, Bellutti M et al. Doubleballoon enteroscopy-assisted virtual chromoendoscopy for small-bowel disorders: a case series. Endoscopy 2009; 41: 468 - 471

\section{Bibliography}

DOI $10.1055 / \mathrm{s}-0030-1256784$

Endoscopy 2012; 44: E101

(c) Georg Thieme Verlag KG Stuttgart · New York ISSN 0013-726X

\section{Corresponding author}

Helmut Neumann, MD, PhD

Department of Medicine I

University of Erlangen-Nuremberg

Ulmenweg 18

91054 Erlangen

Germany

Fax: +49-9131-8535209

helmut.neumann@uk-erlangen.de

Fig. 1 a High-resolution white-light endoscopy revealed pale mucosa with intermittent erythematous eroded patches within the duodenum. b Narrow-band imaging enhanced intestinal villi, which appeared edematous and slightly flattened. Changes in the microvasculature were not visible. c Histopathological examination showed multiple periodic acid-Schiff (PAS)-positive macrophages in the lamina propria. 